\title{
Using Inter-feature-Line Consistencies for Sequence-Based Object Recognition
}

\author{
Jiun-Hung Chen ${ }^{1 \star}$ and Chu-Song Chen ${ }^{2}$ \\ 1 University of Washington, Seattle, WA 98195, USA, \\ jhchen@cs. washington. edu, \\ 2 Institute of Information Science, Academia Sinica, Taipei, Taiwan \\ song@iis.sinica.edu.tw
}

\begin{abstract}
An image sequence-based framework for appearance-based object recognition is proposed in this paper. Compared with the methods of using a single view for object recognition, inter-frame consistencies can be exploited in a sequence-based method, so that a better recognition performance can be achieved. We use the nearest feature line method (NFL) 8] to model each object. The NFL method is extended in this paper by further integrating motion-continuity information between features lines in a probabilistic framework. The associated recognition task is formulated as maximizing an a posteriori probability measure. The recognition problem is then further transformed to a shortest-path searching problem, and a dynamic-programming technique is used to solve it.
\end{abstract}

\section{Introduction}

Appearance-based methods [2] 9 10 12 13] 14, 15] 19 emphasize the use of view-based representations of objects, which are constructed from a set of views of an object in a pre-processing (or learning) stage, for object recognition or tracking. The collection of views is usually recorded in a compact way through principle component analysis (PCA) [10]12], support vector machine (SVM) [13] 14 or neural networks [15] 19. In the past, Murase and Nayar [10 observed that all the training feature vectors (e.g., vectors in association with a PCA representation) of an object consist of a manifold in the feature space. They approximated the manifold by using a spline interpolation for the feature vectors of a set of sampled views. In addition, Roobaert and van Hulle 14 used SVM and Roth, Yang and Ahuja [15] used sparse network of winnows (SNoW) for modelling the sampled views. Appearance-based techniques can also be used for recognizing objects in a cluttered environment [12] 13] and for tracking long image sequences or sequences across views [2].

Object recognition via linear combination [16] 8] [17] is an interesting and informative concept received considerable attentions in recent years. In [16], Ullman and Basri demonstrated that the variety of views depicting the same object

\footnotetext{
* This work was done while he was a research assistant at Institute of Information Science, Academia, Taipei, Taiwan.
} 
under different transformations can often be expressed as the linear combination of a small number of views, and suggested how this linear combination property may be used in the recognition process. In [17, Vetter and Poggio proposed a method based on linear object classes for synthesizing new images of an object from a single $2 \mathrm{D}$ view of the object by using corresponding feature points. In addition to recognizing or synthesizing views of different poses, linear combination has also been shown very useful for visual recognition or view synthesis under different illumination conditions. For example, Belhumeur and Kriegman [1] have shown that a new image under all possible illumination conditions can be expressed as a linear combination of some basis images formed by a convex polyhedral cone in $R^{n}$ if the illumination model is Lambertian.

Recently, a linear method called the nearest feature line (NFL) was proposed for object recognition [8] [7]. It uses the collection of lines passing through each pair of the feature vectors belonging to an object to model appearances of this object. Instead of using splines [10], the NFL method uses a linear structure to represent the appearance manifold, which has a close relationship with the linearcombination approaches mentioned above. In essence, infinite feature vectors of the object class can be generated from finite sample vectors with the NFL method. Note that NFL can be treated as an extension of the nearest-neighbor (NN) method, and it has been theoretically proven that the NFL method can achieve a lower probabilistic error than NN when the number of available feature points for each object class is finite and the dimension of a feature space is high [20]. An experimental evaluation of the NFL method in image classification and retrieval was given in [7], which shows that it can make efficient use of knowledge about multiple prototypes of a class to represent that class.

In this paper, a framework for sequence-based object recognition is proposed by employing the concept of feature lines. In particular, by further considering inter-feature-line consistencies, our method can use several images of an object as the training input for building an image-sequence-based recognition system. More specifically, the database built in our work contains information about possible moves between views in the database. Therefore, it contains much more information than an unordered set of views, and our method would only be applicable to problem domains where this information is available. The main idea of our method is that it tries and finds objects that not only match the individual images, but also makes sure that the sequence of views in the query could match a similar sequence of views in the database. In other words, the input database does not consist of isolated example images; but rather that these images are related to each other via motion consistency. Hence, our method would work much better as more images are added, which means that the performance improvement over adding more images would be relatively better for this method than for other methods. In our framework, a recognition task is formulated as a problem of maximizing an a posteriori probability measure. This problem is further reduced to a most-probable-path searching problem in a specially designed graph, which can be effectively solved with dynamic programming.

This paper presents a general framework for sequence-based object recognition, which can be used for real-world applications such as face recognition. 
For example, by incorporating our recognition method with existing face detection and tracking algorithms [4], 18, this framework can be used to achieve sequence-based face recognition for person identity verification. The remainder of this paper is organized as follows. In Section 2, we present a probabilistic formulation for sequence-based object recognition by employing inter-feature-line consistencies. Section 3 gives the main algorithm of this paper. Some experimental results are shown and discussed in Section 4. Finally, we make conclusions in Section 5.

\section{Object Recognition by Using Inter-feature-Line Consistency}

In the following, we will first review the nearest-feature-line (NFL) method [7] [8] in Section 2.A. Then, we will characterize inter-feature-line consistencies formulated in our work in Section 2.B.

\subsection{Approximate Manifold with Feature Lines - An Introduction}

Assume that we have $M$ objects, and let $X^{c}=\left\{\mathbf{x}_{i}^{c} \mid i=1, \ldots, N_{c}\right\}$ be a set of $N_{c}$ training feature vectors belonging to object $c, c=1,2, \ldots, M$. A feature line $(\mathrm{FL}) \overline{\mathbf{x}_{i}^{c} \mathbf{x}_{j}^{c}}(i \neq j)$ of object $c$ is defined as a straight line passing through $\mathbf{x}_{i}^{c}$ and $\mathbf{x}_{j}^{c}$. A FL space of object $c$ is denoted by $S^{c}=\left\{\overline{\mathbf{x}_{i}^{c} \mathbf{x}_{j}^{c}} \mid 1 \leq i, j \leq N_{c}, i \neq j\right\}$, where $\overline{\mathbf{x}_{i}^{c} \mathbf{x}_{j}^{c}}=\overline{\mathbf{x}_{j}^{c} \mathbf{x}_{i}^{c}}, 1 \leq i, j \leq N_{c}$, and the number of feature lines in $S^{c}$, denoted by $K_{c}$, is $\frac{N_{c}\left(N_{c}-1\right)}{2}$. When there are $M$ classes in the database, $M$ such FL spaces can therefore be constructed, composed of a total number of $N_{\text {total }}=\sum_{c=1}^{M} K_{c}$ FLs. Let $\Gamma=S^{1} \cup S^{2} \cup \ldots \cup S^{M}$ be the collection of all $N_{\text {total }}$ feature lines. The $F L$ distance from a query $\mathbf{q}$ to some feature line $\overline{\mathbf{x}_{i} \mathbf{x}_{j}}(i \neq j)$ is defined as

$$
d\left(\mathbf{q}, \overline{\mathbf{x}_{i} \mathbf{x}_{j}}\right)=\|\mathbf{q}-\mathbf{p}\|
$$

where $\|$.$\| is the 2-norm and \mathbf{p}$ is the projection point of the query $\mathbf{q}$ onto $\overline{\mathbf{x}_{i} \mathbf{x}_{j}}$. The projection point $\mathbf{p}$ can be computed as $\mathbf{p}=\mathbf{x}_{i}+\mu\left(\mathbf{x}_{j}-\mathbf{x}_{i}\right)$ and the position parameter $\mu \in R$ is

$$
\mu=\frac{\left(\mathbf{q}-\mathbf{x}_{i}\right)^{t}\left(\mathbf{q}-\mathbf{x}_{i}\right)}{\left(\mathbf{x}_{j}-\mathbf{x}_{i}\right)^{t}\left(\mathbf{x}_{j}-\mathbf{x}_{i}\right)}
$$

Figure 1 (a) illustrates FLs and FL distances. Note that when $0 \leq \mu \leq 1$, $\mathbf{p}$ is an interpolating point between $\mathbf{x}_{i}$ and $\mathbf{x}_{j}$. Otherwise, $\mathbf{p}$ is a extrapolating point either on the $\mathbf{x}_{i}$ side (when $\mu>1$ ) or the $\mathbf{x}_{j}$ side (when $\mu<0$ ). NFL recognizes $\mathbf{q}$ as object $c^{*}$ by computing the minimal FL distance between all features lines contained in $\Gamma$ as shown below.

$$
\begin{aligned}
d\left(\mathbf{q}, \overline{\mathbf{x}_{i^{*}}^{c^{*}} \mathbf{x}_{j^{*}}^{c^{*}}}\right) & =\min _{1 \leq c \leq M} \min _{\mathbf{x}_{i}^{c} \mathbf{x}_{j}^{c} \in S^{c}} d\left(\mathbf{q}, \overline{\mathbf{x}_{i}^{c} \mathbf{x}_{j}^{c}}\right) \\
& =\min _{\overline{\mathbf{x}_{i}^{c} \mathbf{x}_{j}^{c}} \in \Gamma} d\left(\mathbf{q}, \overline{\mathbf{x}_{i}^{c} \mathbf{x}_{j}^{c}}\right)
\end{aligned}
$$






(a)

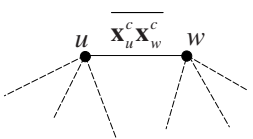

(b)

Fig. 1. (a)The concepts of a FL $\overline{\mathbf{x}_{i} \mathbf{x}_{j}}$ and the FL distance from a query $x$ to $\overline{\mathbf{x}_{i} \mathbf{x}_{j}}$. (b)The concept of neighboring FLs. The feature lines drawn with dash lines are neighboring FLs of $\overline{\mathbf{x}_{u}^{c} \mathbf{x}_{w}^{c}}$

\subsection{Inter Feature-Line Consistencies}

In essence, the NFL method [8] 7] only uses the distance-from-manifold information for object recognition. In our framework, we further extend the NFL method to a new one where inter-feature-line consistencies are incorporated by using the concept of neighboring FLs. Given a feature line $\overline{\mathbf{x}_{u}^{c} \mathbf{x}_{w}^{c}}$, we denote $\Psi_{c, u, w}=\left\{\overline{\mathbf{x}_{u}^{c} \mathbf{x}_{w}^{c}}\right\} \cup\left\{\overline{\mathbf{x}_{u}^{c} \mathbf{x}_{w_{1}}^{c}} \mid 1 \leq w_{1} \leq N_{c}, w_{1} \neq u, w_{1} \neq w\right\} \cup\left\{\overline{\mathbf{x}_{u_{1}}^{c} \mathbf{x}_{w}^{c}} \mid 1 \leq\right.$ $\left.u_{1} \leq N_{c}, u_{1} \neq w, u_{1} \neq u\right\}$ to be the set of its neighboring FLs. An illustration of neighboring FLs is shown in Figure 1(b). The number of neighboring FLs of $\overline{\mathbf{x}_{u}^{c} \mathbf{x}_{w}^{c}}$ is therefore equal to $2 N_{c}-3$.

Let $\Lambda_{\mathbf{q}}=\left\{\mathbf{q}_{0}, \mathbf{q}_{1} \ldots, \mathbf{q}_{L}\right\}$ be a sequence of $L+1$ views. In addition, let $\Psi=\left\{\mathbf{l}_{i} \in \Gamma \mid i=0,1, \ldots, L\right\}$ be a set of $L+1$ FLs where each $\mathbf{q}_{i}$ matches its projection point $\mathbf{p}_{i}$ on $\mathbf{l}_{i}$. We recognize $\Lambda_{\mathbf{q}}$ by finding $\Psi^{*}$ that maximizes the following a posteriori probability:

$$
\begin{aligned}
\Psi^{*} & =\arg \max _{\Psi} P\left(\Psi \mid \Lambda_{\mathbf{q}}\right) \\
& =\arg \max _{\Psi} P\left(\mathbf{l}_{0}, \ldots, \mathbf{l}_{L} \mid \mathbf{q}_{0}, \ldots, \mathbf{q}_{L}\right) \\
& =\arg \max _{\Psi} P\left(\mathbf{q}_{0}, \ldots, \mathbf{q}_{L} \mid \mathbf{l}_{0}, \ldots, \mathbf{l}_{L}\right) P\left(\mathbf{l}_{0}, \ldots, \mathbf{l}_{L}\right) \\
& =\arg \max _{\Psi} P\left(\mathbf{l}_{0}\right) P\left(\mathbf{q}_{0} \mid \mathbf{l}_{0}\right) \prod_{i=0, \ldots, L-1} P\left(\mathbf{l}_{i+1} \mid \mathbf{l}_{i}\right) P\left(\mathbf{q}_{i+1} \mid \mathbf{l}_{i+1}\right),
\end{aligned}
$$

where the last equality holds by assuming that

(i) $P\left(\mathbf{q}_{i} \mid \mathbf{l}_{i}\right), i=0, \ldots, L$ are independent of each other, and

(ii) $P\left(\mathbf{l}_{0}, \ldots, \mathbf{l}_{L}\right)$ can be modelled by a first-order Markov chain. That is, $P\left(\mathbf{l}_{i} \mid \mathbf{l}_{i-1}, \mathbf{l}_{i-2}, \ldots, \mathbf{l}_{0}\right)=P\left(\mathbf{l}_{i} \mid \mathbf{l}_{i-1}\right)$ for all $i=1, \ldots, L$.

To evaluate (4), the transition probabilities between the feature lines $\mathbf{l}_{i}$ and $\mathbf{l}_{i+1}, P\left(\mathbf{l}_{i+1} \mid \mathbf{l}_{i}\right), i=0, \ldots, L-1$, and the likelihoods $P\left(\mathbf{q}_{i} \mid \mathbf{l}_{i}\right), i=0, \ldots, L$, have to be specified. First, we can see that $P\left(\mathbf{q}_{i} \mid \mathbf{l}_{i}\right)$ is a probability measure in association with the similarity between $\mathbf{y}_{i}$ and $\mathbf{q}_{i}$, where $\mathbf{y}_{i}$ is the projection point of $\mathbf{q}_{i}$ onto $\mathbf{l}_{i}$. Hence, $P\left(\mathbf{q}_{i} \mid \mathbf{l}_{i}\right)$ can be set as being decreased with $\left\|\mathbf{q}_{i}-\mathbf{y}_{i}\right\|$, the distance between the observation $\mathbf{q}_{i}$ to its projection point $\mathbf{y}_{i}$. We thus refer this probability to as the probability caused by the distance from appearance manifolds (PDAM). Second, because the image sequence to be recognized consists of consecutive views of an object, $P\left(\mathbf{l}_{i+1} \mid \mathbf{l}_{i}\right)$ is larger when $\mathbf{l}_{i+1}$ is a more reasonable 
consequent of $\mathbf{l}_{i}$ by considering motion continuity. We refer $P\left(\mathbf{l}_{i+1} \mid \mathbf{l}_{i}\right)$ to as the probability caused by motion continuity (PMC). Nevertheless, note that here the concept of "motion continuity" has nothing to do with velocity or rotation, but rather only whether the relationship between images in the database matches the relationship between two images in the query.

\section{Probabilistic Framework and Main Algorithm}

\subsection{Probability Distribution Setting for PDAM and PMC}

To evaluate (4), we make the following assumptions:

First, the PDAM is modelled as a Gaussian distribution, $P\left(\mathbf{q}_{k} \mid \overline{\mathbf{x}_{u}^{c} \mathbf{x}_{w}^{c}}\right)=$ $\exp \left(-d_{c ; u, w ; k}^{2} / 2 \sigma^{2}\right) / Z$, where $\sigma$ is a chosen constant, $Z$ is a normalization constant which normalizes $P\left(\mathbf{q}_{k} \mid \overline{\mathbf{x}_{u}^{c} \mathbf{x}_{w}^{c}}\right)$ to be a probability density function, and

$$
d_{c ; u, w ; k}=d\left(\mathbf{q}_{k}, \overline{\mathbf{x}_{u}^{c} \mathbf{x}_{w}^{c}}\right)
$$

for $k=0, \ldots, L, c=1, \ldots, M, 1 \leq u, w \leq N_{c}$ and $u \neq w$.

Second, let the PMC be defined as follows:

$$
P\left(\mathbf{l}_{i+1} \mid \mathbf{l}_{i}\right)=\left\{\begin{array}{l}
0 ; \text { If } \mathbf{l}_{i+1} \text { is not a neighbor of } \mathbf{l}_{i} \\
\frac{1}{N\left(\mathbf{l}_{i}\right)} ; \text { Otherwise. }
\end{array}\right.
$$

where $N\left(\mathbf{l}_{i}\right)=2 N_{c}-3$ is the number of neighboring FLs for $\mathbf{l}_{i}$.

Third, we assume equal priori probabilities for all the FLs by setting $P(\mathbf{l})=$ $1 / N_{\text {total }}$ for $\mathbf{l} \in \Gamma$.

Taking a natural log of (4), the following formulation can be derived:

$$
\begin{aligned}
\Psi^{*} & =\arg \max _{\Psi} \ln \left(P\left(\mathbf{l}_{0}\right) P\left(\mathbf{q}_{0} \mid \mathbf{l}_{0}\right) \prod_{i=0, \ldots, L-1} P\left(\mathbf{l}_{i+1} \mid \mathbf{l}_{i}\right) P\left(\mathbf{q}_{i+1} \mid \mathbf{l}_{i+1}\right)\right) \\
& =\arg \min _{\Psi} \ln J\left(\Psi ; \Lambda_{\mathbf{q}}\right), \\
& =\arg \min _{\Psi} J\left(\Psi ; \Lambda_{\mathbf{q}}\right),
\end{aligned}
$$

where

$$
J\left(\Psi ; \Lambda_{\mathbf{q}}\right) \mathbf{1}=\left\{\begin{array}{l}
\infty \text { if } P\left(\mathbf{l}_{i+1} \mid \mathbf{l}_{i}\right)=0 ; \quad \text { for some } i \in\{0, \ldots, L-1\}, \\
\sum_{i=0}^{L} d\left(\mathbf{q}_{i}, \mathbf{l}_{i}\right)^{2} / 2 \sigma^{2}-\sum_{i=0}^{L-1} \ln \left(2 N_{c}-3\right)-\ln N_{\text {total }} ; \quad \text { Otherwise } .
\end{array}\right.
$$

From (7), to find $\Psi^{*}$ that maximizes the a posteriori probability is equivalent to find $\Psi^{*}$ that minimizes the objective function $J$ defined in (8). Note that it is computationally intractable to use brute force for computing $\Psi^{*}$. In this work, the PDAM and PMC are encoded in a matching graph, and dynamic programming is adopted for solving this minimization problem. 


\subsection{Construction of Matching Graph}

We construct a graph $G$ containing $L+1$ levels. There are $N_{\text {total }}$ nodes in each level, where $N_{\text {total }}$ is the total number of feature lines defined before. For the $k$-th level, $(k=0, \ldots, L)$, a number of $N_{\text {total }}$ nodes, denoted by $n_{\overline{\mathbf{x}_{u}^{c} \mathbf{x}_{w}^{c}} ; k}$, $c \in\{1,2, \ldots, M\}$ and $u, w \in\left\{1,2, \ldots, N_{c}\right\}$, are constructed in association with it. In addition to these nodes, we also construct a source node $n_{-1}$ and a sink node $n_{L+2}$. Then, some edges are constructed by connecting nodes in $G$ as follows. The source node $n_{-1}$ and sink node $n_{L+2}$ are fully connected to nodes at level 1 and level $L+1$, respectively. For each adjacent levels $k$ and $k+1$, $k=0, \ldots, L-1$, there is an edge $e\left(\overline{\mathbf{x}_{u_{1}}^{c} \mathbf{x}_{w_{1}}^{c}} ; \overline{\mathbf{x}_{u_{2}}^{c} \mathbf{x}_{w_{2}}^{c}} ; k\right)$ linking $n_{\overline{\mathbf{x}_{u_{1}}^{c} \mathbf{x}_{w_{1}}^{c}} ; k}$ to $n \overline{\mathbf{x}_{u_{2}}^{c} \mathbf{x}_{w_{2}}^{c}} ; k+1$ if $\overline{\mathbf{x}_{u_{1}}^{c} \mathbf{x}_{w_{1}}^{c}} \in \Psi_{c, u_{2}, w_{2}}$, the set of neighbor FLs of $\overline{\mathbf{x}_{u_{2}}^{c} \mathbf{x}_{w_{2}}^{c}}$. Figure 2 shows an illustration of the graph $G$ in association with the case in which there are two objects and each object has four views.

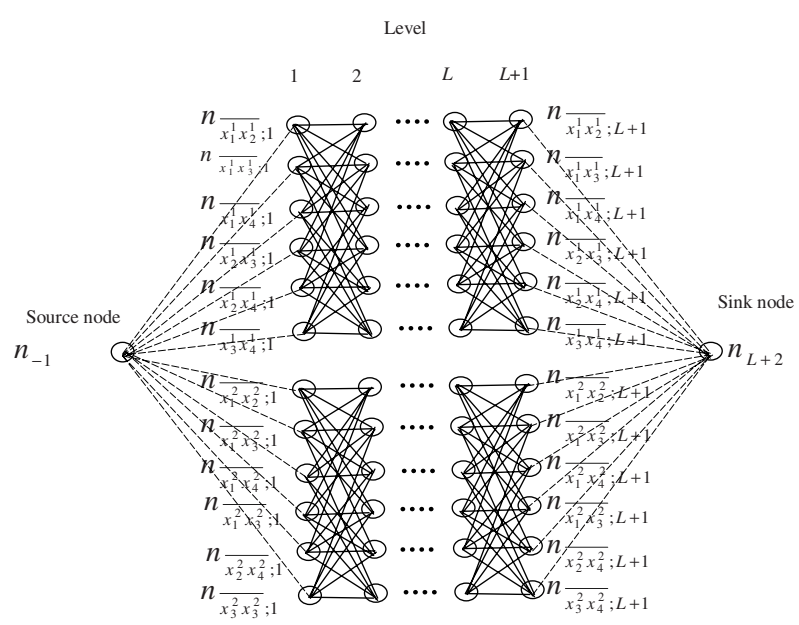

Fig. 2. An example of a graph $G$ that is constructed for the case of two objects, where each object contains four views. There are therefore $6=C_{2}^{4}$ FLs for each object

For each node $n_{\overline{\mathbf{x}_{u}^{c} \mathbf{x}_{w}^{c} ; k}}$ (except the source and sink nodes), a node score $s_{c ; u, w ; k}$ is assigned to it by setting

$$
s_{c ; u, w ; k}=d_{c ; u, w ; k}^{2} / \sigma^{2}
$$

This node score is used to encode the log likelihood of the PDAM in (8). In addition, the scores of the source and sink nodes, $s_{-1}$ and $s_{L+2}$, are both set to 0 .

Then, the cost of a node is defined from the principle of dynamic programming as shown in the following. First, the cost of the source node, $\operatorname{cost}\left(n_{-1}\right)$, is set to zero. Then, the cost of each of the other nodes is defined recursively as

$$
\operatorname{cost}\left(n_{\overline{\mathbf{x}_{u}^{c} \mathbf{x}_{w}^{c} ; k}}\right)=s_{c ; u ; w ; k}+\min \left\{\operatorname{cost}\left(n_{\overline{\mathbf{x}_{u^{\prime}}^{c} \mathbf{x}_{w^{\prime}}^{c} ; k^{\prime}}}\right) \mid n_{\overline{\mathbf{x}_{u^{\prime}}^{c} \mathbf{x}_{w^{\prime}}^{c} ; k^{\prime}}} \in \Theta_{c ; u ; w ; k}\right\} ;
$$

for $k=1, \ldots, L+2, c=0, \ldots, M$ and $0 \leq u, w \leq N_{c}$. Note that $\Theta_{c ; u ; w ; k}$ is the set of nodes having edges linking to $n \overline{\mathbf{x}_{u}^{c} \mathbf{x}_{w}^{c}} ; k$. 


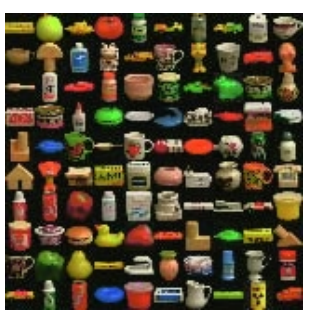

(a)

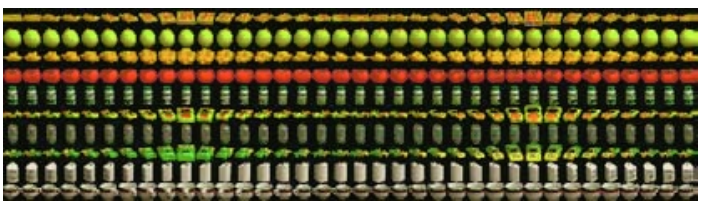

(b)

Fig. 3. Coil-100 data set. (a) The 100 objects in the Coil-100 data set. (b) Some views of ten of the objects shown in (a) (sampled by every $10^{\circ}$ )

Each path starting from the source node and ending at the sink node represents a sequence of matches between a view belonging to $\Lambda_{\mathbf{q}}$ and a FL belonging to $\Gamma$. The cost of the sink node, $\operatorname{cost}\left(n_{L+2}\right)$, is then referred to as the minimal cost, and it is easy to verify that $\operatorname{cost}\left(n_{L+2}\right)=\min _{\Psi} J\left(\Psi ; \Lambda_{\mathbf{q}}\right)$ defined in (8)). The path associated with the minimal cost or, equivalently, the shortest path from the source to the sink nodes, is then referred to as the matching (or optimal) path in this work. The FL associated with nodes in $G$ (except the source and sink nodes) are then treated as a sequence of matched FLs of the sequence of testing consecutive views, $\Lambda_{\mathbf{q}}$, and the object represented by these FLs then serves as the recognition result.

In our work, to avoid recursive programming, the Dijkstra algorithm 5] is used to find the optimal path. For each node, an incoming edge with the lowest accumulated cost is retained in our approach. After finding the best incoming choice for all nodes, our process backtracks, from the sink to the source nodes, to obtain the optimal path. Except for the source and sink nodes, each node passed by the optimal path then represents a match between a view belonging to $\Lambda_{\mathbf{q}}$ and a FL belonging to $\Gamma$.

\section{Experimental Results and Discussions}

\subsection{Experimental Results}

Coil-100 Object Recognition. The Coil-100 data set 11 was widely used as an object-recognition benchmark [10] 14] 15]. In this data set, there are 100 objects and each object has 72 different views (images) that are taken every $5^{\circ}$ around an axis passing through the object. Each image is a 128x128 color one with R,G,B channels. Figures [3(a) and 3(b) show these 100 objects and some sampled views of ten of these objects, respectively.

We follow the experimental settings in [15], which used only a limited number of views per objects for training. In our experiment, four different views per object $\left(0^{\circ}, 90^{\circ}, 180^{\circ}\right.$ and $\left.270^{\circ}\right)$ were used for training, as shown in Figure 4(a), and the other 6800 (i.e., $\left.(72-4)^{*} 100=6800\right)$ images were used for testing. In other words, for each object, 6 features lines can be constructed from its 4 training views. 


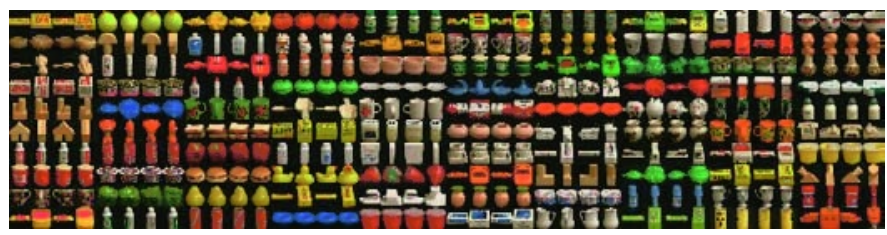

(a)

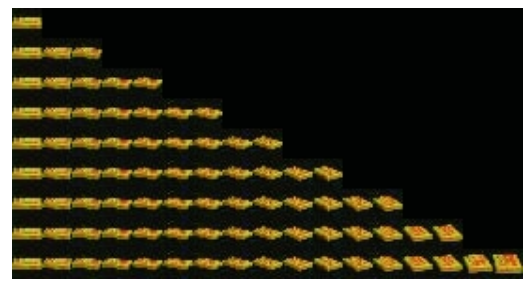

(b)

Fig. 4. (a) All the training views used in the experiments. (b) The sequences of views of the first object used in our experiment (with the lengths being 1 to 17)

In our experiment, the number of consecutive views (i.e., $L+1$ ) used for testing was set to be $1,3,5, \ldots, 17$. A sequence of consecutive testing views of an object are shown in Figure 4 (b). Note that when the number of consecutive testing views is 1 , our method degenerates to the NFL method with only a single image as its input. For each number of consecutive testing views, the average recognition error rate over 6800 tests was computed and recorded. Note that the sets of training and testing views are disjoint in our experiment, and thus the training views were dropped out when an image sequence was sampled for testing.

In the first test, the input image format was set to be colored $16 \times 16$, which is the same as that in [14, and the 16x16 image directly serves as the feature vector. Figure 5 shows the experimental results by using our method. From this figure, it can be seen that our method can considerably improve the recognition performance of the NFL method as the number of consecutive testing views is increased. To further show that our probabilistic framework can integrate the consecutive visual clues better, two additional methods, NN-voting and NFL-voting, which are simply extended from the nearest-neighbor and nearestfeature-line methods to those employing image sequences by majority voting (i.e., the identity of recognition is determined by which receiving the maximal number of votes when every view in the sequence is independently recognized), respectively. As shown in Figure 5, our method outperform either NN-voting or NFL-voting because appearance-similarity and motion-continuity information is appropriately exploited.

In the second test, we investigate how the recognition error rates of our method can improve as more training images were added. In this test, $32 \times 32$ gray images from the same database were used, as those adopted in [15, and the $32 \times 32$ image directly serves as the feature vector. We vary the number of training views from 4 to 8 and Figure 6 shows these results. For example, when 


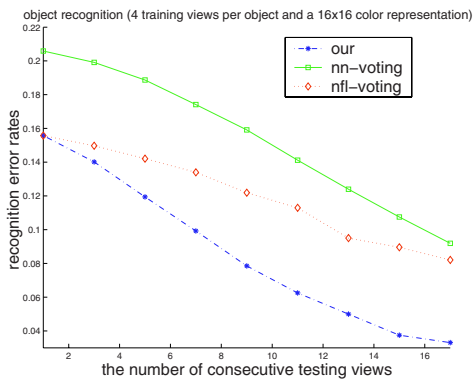

Fig. 5. Object recognition results using the same image format (i.e., color 16x16 images) as that in 14. We compare our method (our) with nearest neighbor voting (nn-voting) and nearest feature line voting (nfl-voting) methods

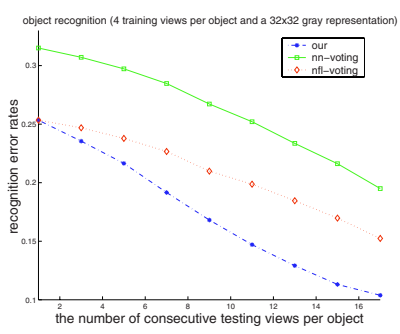

(a)

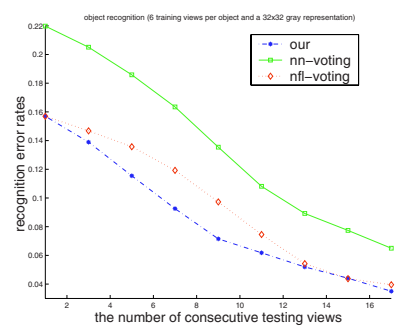

(b)

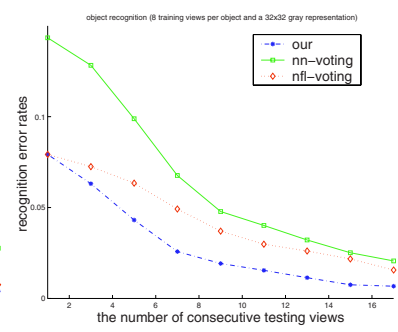

(c)

Fig. 6. Object recognition results using the same image format (i.e., gray level $32 \times 32$ images) as that in [15.(a) 4 training views. (b) 6 training views (c) 8 training views

the numbers of training examples (FLs) increase from 4 (6) to 6 (15) and 8 (28), the lowest recognition error rates decrease from $10.38 \%$ to $3.5 \%$ and $0.67 \%$, respectively. From these experimental results, it is shown that the performance of the method would dramatically improve as more images are added. This figure also shows that our method can greatly improve the single-view-based NFL method and are better than the NN-voting and NFL-voting methods.

Face Recognition. We perform face recognition on a face-only database 2 . There are 1280 image of 128 persons, where each person has 10 images with distinct poses or expressions per person. Each image size is normalized to be $32 \times 32$, which directly serves as the features being used. Figures 7 (a) and (b) show all 128 persons and all 10 views of the first 5 persons in this face database. In the following experiments, we use 5 different views (that is, $15 \mathrm{FLs}$ ) per person in training as shown in Figure 7(c) (left part), and other (i.e., (10-5)*128=640) images are used for testing. Hence, for each object, 15 features lines can be constructed from its 5 training views. In addition, the number of consecutive testing views is sampled from $\{1,3,5\}$. Some examples of sequences of three views are shown in Figure 7 (c) (right part).

\footnotetext{
${ }^{2}$ This database can be download from http://smart.iis.sinica.edu.tw/html/face.html
} 


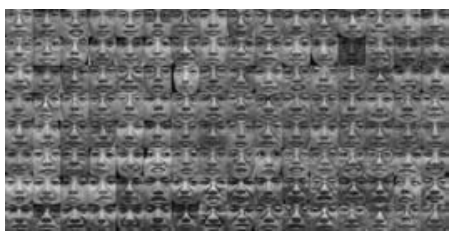

(a)

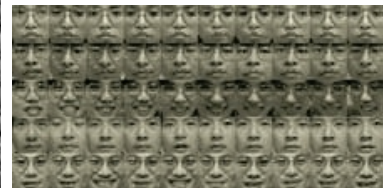

(b)

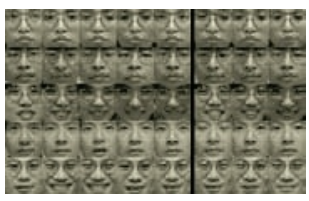

(c)

Fig. 7. A face-only database. (a) shows all 128 persons in this database. (b) shows all 10 views of the first 5 persons shown in the first row of (a) . (c) shows training views (left part) and some examples of sequences of three consecutive testing views of these 5 persons (right part), respectively. Note that the training views are formed by collecting faces in columns 1, 3, 5, 7 and 9 shown in (b).

Table 1 shows the experimental results using our method and the comparisons between our method and NN-voting and NFL-voting. In addition, it also shows that our method give the best performance.

Table 1. Performance comparison between our method (our) with nearest neighbor voting (nn-voting) and nearest feature line voting (nfl-voting) methods on the faceonly database. Recognition error rates in $\%$ on different numbers of consecutive testing views are shown. Our method gives the best performance.

\begin{tabular}{|l|c|c|c|}
\hline & Ours & NN-Voting & NFL-Voting \\
\hline number of testing views (1) & 5.31 & 6.71 & 5.31 \\
\hline number of testing views (3) & 0.26 & 2.86 & 2.6 \\
\hline number of testing views (5) & 0 & 1.55 & 1.56 \\
\hline
\end{tabular}

\subsection{Discussions}

To our best knowledge, no image-sequence-based methods have been used for testing these three databases in the past, and some existing results are introduced in the following for comparison.

For Coil-100 database, the following results have been reported. When the image format is colored $16 \times 16$, and the number of training views is four, the PCA-and-Spline-Manifold [10] and the linear SVM method [14] achieved $12.4 \%$ and $13.1 \%$ recognition error rates, respectively (the NFL method achieved $15.6 \%$ in our testing). When the image format is 32x32 gray, the linear SVM method [14] and the snow-with image method [15] achieve $18.4 \%$ and $21.5 \%$ error rates, respectively (the NFL method achieved 25.3\% in our testing). From Figures 5 and 6(a), it can been seen that our method can outperform the existing results shown above when only 5 and 9 consecutive images of objects are used for colored $16 \times 16$ and $32 \times 32$ gray image formats (four training views), respectively. ${ }^{3}$ For the face-only face database, $13 \%$ recognition error rate can be achieved in [3] when

\footnotetext{
3 The above results were all tested based purely on image intensity information. Roth, Yang and Ahuja [15] have further exploited edge information to achieve a better
} 
their method is tested on a part of this database (100 persons) and 6 images per person are used as training images. From Table 1, it can been seen that our method has lower recognition error rates even when only four training views are used. The results reveal that the recognition performance can be considerably upgraded by appropriately exploiting useful visual clues contained in an image sequence.

Some further remarks are addressed below:

Remark 1 [Neighborhood Relationships]: Although each of our experiments uses a database with a single linear sequence of views as the object rotates about a single axis, other kinds of neighborhood relationships in the database could be exploited. For example, our approach can also be used for the case that a database contains views as the object is rotated about two axes [6]. In this case, the neighborhood relationship is two dimensional but not one dimensional, and our approach is still applicable for this case.

Remark 2 [Feature Selection]: Although raw data was directly used as feature in our experiment, this is not the only choice. Our method can also use features produced by feature-extraction or feature-generation processes such as principal component analysis or linear discriminant analysis, and features generated in such ways would have chance to be helpful for either the computational efficiencies or the recognition accuracies.

\section{Conclusions}

There are several characteristics of our framework for appearance-based object recognition using a sequence of views. First, we emphasize inter-feature-line consistencies. Second, we take both the probability caused by the distance from manifold, PDAM, and the probability caused by motion continuity, PMC, into considerations. Third, to handle the associated recognition problem, we construct a matching graph in which PDAM and PMC are incorporated, and transform this problem into a shortest path problem that can be effectively solved by using dynamic programming. The experimental results on the Coil-100 data set and the face-only database show that our method achieves high recognition rates for object recognition and face recognition. Our method thus provides an effective way for appearance-based object recognition using a sequence of views.

Acknowledgments. This work is supported in part by the National Science Council of Taiwan under Grant NSC project, NSC 91-2213-E-001-022. J.-H. Chen acknowledges travel support from Department of Computer Science and Engineering, University of Washington at Seattle.

error rate, $11.7 \%$, in their snow-with-image-and-edge method. Our result is better when the number of views in use is 15 without using edge information as shown in Figure 5 (four training views). 


\section{References}

1. P. N. Belhumeur and D. J. Kriegman "What is the Set of Images of an Object under all Possible Illumination Conditions?," International Journal of Computer Vision, vol. 28, pp. 245-260, 1998.

2. M. J. Black and A. Jepson, "EigenTracking: Robust Matching and Tracking of Articulated Objects Using a View-Based Representation," International Journal of Computer Vision, vol. 26, pp. 63-84, 1998.

3. L.-F. Chen, H.-Y. M. Liao, M.-T. Ko, J.-C. Lin, and G.-J. Yu "A New LDAbased Face Recognition System Which Can Solve the Small Sample Size Problem," Pattern Recognition, vol. 33, pp. 1713-1726, 2000.

4. Y.-S. Chen, and et al. "Video-based Eye Tracking for Autostereoscopic Displays," Optical Engineering, vol. 40, pp. 2726-2734, 2001.

5. T. H. Cormen, C. E. Leiserson, and R. L. Rivest, Introduction to Algorithms, MIT Press, Cambridge, MA, 1990.

6. Y.-P. Hung, C.-S. Chen, Y.-P. Tsai, S.-W. Lin, "Augmenting Panoramas with Object Movies by Generating Novel Views with Disparity-Based View Morphing," Journal of Visualization and Computer Animation, vol. 13, pp. 237-247, 2002.

7. S.Z. Li, K.L. Chan and C.L. Wang, "Performance Evaluation of the Nearest Feature Line Method in Image Classification and Retrieval," IEEE Transactions on Pattern Analysis and Machine Intelligence, vol. 22, pp. 1335-1339, 2000.

8. S.Z. Li and J. Lu, "Face Recognition Using the Nearest Feature Line Method," IEEE Transactions on Neural Networks, vol. 10, pp. 439-443, 1999.

9. B. Moghaddam and A. Pentland, "Probabilistic Visual Learning for Object Representation," IEEE Transactions on Pattern Analysis and Machine Intelligence, vol. 19, pp. 696-710, 1997.

10. H. Murase and S. K. Nayar, "Visual Learning and Recognition of 3D Objects from Appearance," International Journal of Computer Vision, vol. 14, pp. 5-24, 1995.

11. S. A. Nene, S. K. Nayar and H. Murase, "Columbia Object Image Library (COIL100)," Technical Report CUCS-006-96, Columbia University, 1996.

12. K. Ohba and K. Ikeuchi, "Detectability, Uniqueness, and Reliability of Eigen Widows for Stable Verification of Partially Occluded Objects," IEEE Transactions on Pattern Analysis and Machine Intelligence, vol. 19, pp. 1043-1048, 1997.

13. C. Papageorgious and T. Poggio, "A Pattern Classification Approach to Dynamic Object Detection," in Proceedings of International Conference on Computer Vision, Corfu, Greece, 1999, pp. 1223-1228.

14. D. Roobaert and M. M. van Hulle, "View-based 3D Object Recognition with Support Vector Machines," in Proceedings of 1999 IEEE International Workshop on Neural Networks for Signal Processing, pp. 77-84, Madison, Wisconsin, USA, 1999.

15. D. Roth, M.-H. Yang and N. Ahuja, "Learning to Recognize 3D Objects," Neural Computation, vol. 14, pp. 1071-1103, 2002.

16. S. Ullman and R. Basri, "Recognition by Linear Combinations of Models," IEEE Transactions on Pattern Analysis and Machine Intelligence, vol. 13, pp. 992-1006, 1991.

17. T. Vetter and T. Poggio, "Linear Object Classes and Image Synthesis From a Single Example Image," IEEE Transactions on Pattern Analysis and Machine Intelligence, vol. 19, pp. 696-710, 1997.

18. P. Viola and M. Jones, "Rapid Object Detection using a Boosted Cascade of Simple Features," in IEEE Conference on Computer Vision and Pattern Recognition, pp. 511-518, Kauai, Hawaii, USA, 2001. 
19. C. Yuan and H. Niemann, "An Appearance Based Neural Image Processing for 3-D Object Recognition," in Proceedings of International Conference on Image Processing, Vancouver, Canada, pp. 344-347, 2000.

20. Z. Zhou, S. Z. Li and K. L. Chan, "A Theoretical Justification of Nearest Feature Line Method," in Proceedings of International Conference on Pattern Recognition, Barcelona, Spain, pp. 759-762, 2000. 